\title{
Secondary light-ion transport from intermediate-energy hadron experiments
}

\author{
Ashwin P. Srikrishna ${ }^{1, \mathrm{a}}$, Luis A. Castellanos ${ }^{1}$, Natalie A. McGirl ${ }^{1}$, Lawrence H. Heilbronn ${ }^{1}$, Chiara La Tessa ${ }^{2}$, Adam \\ Rusek $^{2}$, Michael Sivertz ${ }^{2}$, Steve Blattnig ${ }^{3}$, Martha Clowdsley ${ }^{3}$, Tony Slaba ${ }^{3}$, and Cary Zeitlin ${ }^{4}$ \\ ${ }^{1}$ Department of Nuclear Engineering, University of Tennessee, 1004 Estabrook Rd, Knoxville, TN, USA, 37996 \\ ${ }^{2}$ NASA Space Radiation Laboratory, Brookhaven National Laboratory, Upton, NY, USA, 11973 \\ ${ }^{3}$ National Aeronautics and Space Administration, Langley Research Center, Hampton, VA, USA, 23681 \\ ${ }^{4}$ Lockheed Martin, Information Systems and Global Solutions, Houston, TX, USA, 77285
}

\begin{abstract}
The aim of this research is to produce double differential thick target yields, angular distributions and integrated yields for the inclusive production of neutrons, protons, deuterons, tritons, ${ }^{3} \mathrm{He}$, and ${ }^{4} \mathrm{He}$ from intermediate heavy-ion interactions on thick targets of aluminium, polyethylene and other targets of interest to the radiation shielding program as specified by the National Aeronautics and Space Administration (NASA). In tandem with the experimental research, transport model calculations of these thick target yields were also performed. The first such experimental run was conducted in May 2015, with the expectation of improved experimental results at a following March 2016 run at the NASA Space Radiation Laboratory (NSRL) on the campus of Brookhaven National Laboratory (BNL). The May 2015 commissioning run served to test the electronics of the experimental setup, as well as the various detectors and other equipment under the conditions in which the following measurements will be run. The series of future acceleratorbased experiments will rely on the inclusion of two separate upstream and downstream targets. Analysis of the data from both sets of detectors - liquid scintillator and sodium iodide - using both pulse height and time-of-flight methods will allow NASA to perform uncertainty quantification and sensitivity analysis on their transport codes and future shielding studies.
\end{abstract}

\section{Introduction}

There are two primary sources of ionizing radiation in the solar system - solar energetic particles (SEP) and galactic cosmic rays (GCR). Any future manned mission into space requires a more complete understanding of the radiation effects of exposure to these two types of ionizing radiation. SEP derive from energy released via solar flares in the form of highly energetic nuclei, so an increase in the frequency or intensity of solar flares (i.e. mimicking the solar activity cycle) roughly corresponds with an increase in SEP flux [1]. And similarly, GCR derive from the ever-present high energy nuclei present in the cosmos. Naturally, dose reduction is a major target for the future success of deep-space missions, and the current strategies rely on the use of thick (areal density of $30 \mathrm{~g} / \mathrm{cm}^{2}$ or greater) shielding materials, including aluminium and high density polyethylene [1]. NASA is attempting to model these types of shielding setups through the use of both stochastic Monte Carlo codes (FLUKA, PHITS, etc.) and deterministic codes (HZETRN), but there are issues involving both code accuracy and computational runtime [2]. Running experiments designed to calculate double differential (spatial, energy) thick target yields at the NASA Space Radiation Laboratory (NSRL) at Brookhaven National Laboratory (BNL) will allow for a collaboration with NASA to improve the quality of the shielding codes used by NASA. These results will allow for the opportunity to practically study the output of these simulations, as well as provide input in the form of uncertainty quantification and sensitivity analysis in the development of NASA codes.

Figure 1 illustrates one such example of the various models (HZETRN, Geant4, FLUKA and PHITS) providing very interesting results from a simulation of a GCR field spectrum directed at a water target with both front and back shields of infinite lateral dimensions [2]. There are differences between the models, but all seem to imply that at a particular point, increasing the shielding actually increases the calculated dose equivalent. Figure 1 represents the dose equivalent from all charged particles and charged ions - but if specific segments are looked at, the specific deviations can be found. For instance, looking only at the differential flux for deuterons, tritons, helium-3 and helium- 4 in this simulated setup, there are statistically-significant disagreements amongst all of the codes. For the heavier ions $(Z>8)$ and heavy ion fragments $(2<Z<8)$, there are reasonable levels of agreement on dose contributions. As such, this experiment is designed to study the $Z=1 \& 2$ particles - their production, their various energy- and spatial-based fluxes, and their dose contributions inside the target area.

\footnotetext{
a Corresponding author: asrikris@vols.utk.edu
} 


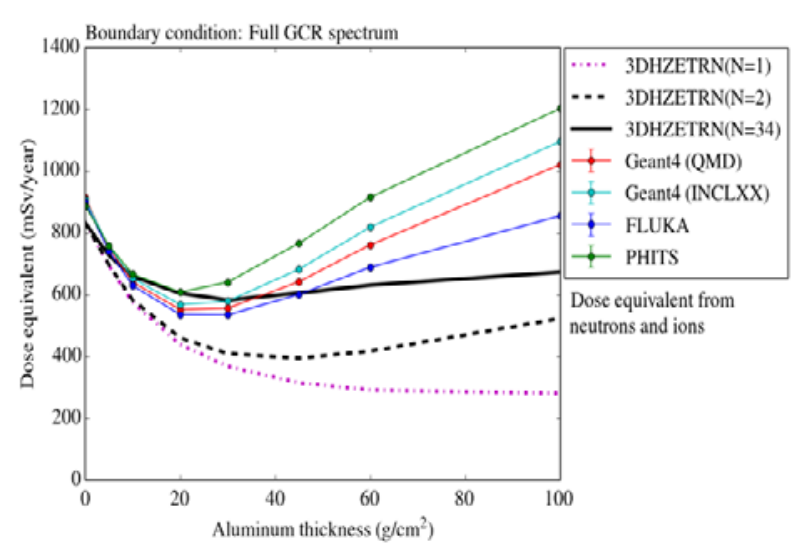

Figure 1. NASA code output for a simulated water target with front and back shielding struck by a full GCR spectrum [2].

This seems to suggest that at a point, while the shielding decreases the exposure concerns with respect to the primary GCR and SEP radiation fields, a secondary radiation field is created posing an additional risk [3]. This secondary radiation field - composed primarily of neutrons and charged light ions - is derived from the primary ionizing radiation interacting with the shielding material. This is further exacerbated by the secondary field interacting with the back (downstream) target and producing a tertiary field. The highly penetrative nature and large dose equivalent factors of the secondary radiation field necessitate that a better understanding of this field is needed for both the safety of the astronauts and the improvement of these transport codes $[2,4]$. This experiment is designed to study and investigate these light ions by simulating the effects of GCR and SEP on varying aluminium and HDPE shielding thicknesses.

\section{Experiment Design}

Secondary light ion production (including neutrons and charged light ions ranging from protons up through ${ }^{4} \mathrm{He}$ ) were measured during a commissioning run at the NSRL facility at BNL in May of 2015. The experiment focused on four separate beams -400 and $2500 \mathrm{MeV}$ protons, and $400 \mathrm{MeV} /$ nucleon and $1000 \mathrm{MeV} /$ nucleon iron striking a single upstream target of $30 \mathrm{~g} / \mathrm{cm}^{2}$ aluminum

The sodium iodide array was composed of four $4 \mathrm{x}$ $2 \times 16$ in $^{3}\left(10.16 \times 5.08 \times 60.64 \mathrm{~cm}^{3}\right)$ sodium iodide detectors. These detectors were the best available at the time of the commissioning run, and have since been replaced by larger $4 \times 4 \times 16$ in $^{3}(10.16 \times 10.16 \times 60.64$ $\mathrm{cm}^{3}$ ) sodium iodide detectors provided by the Department of Homeland Security on long-term loan to the University of Tennessee (UT-Knoxville). These detectors originate from portal monitors no longer in use, and allow for the implementation of additional sodium iodide arrays. They are twice as thick as the detectors used in the commissioning run, meaning only two such detectors are necessary to form a similarly sized array. The new detector setup for the March 2016 experimental run can be seen in Figure 2.
The locations for the organic liquid scintillators (ELJEN Model EJ-301) has slightly changed (each arc represents a radial increase of $0.5 \mathrm{~m}$ ), due to the inclusion of more sodium iodide arrays, as well as the introduction of shadow bars. The large sections of iron shadow bars (lengths of $1 \mathrm{~m}$ and $2 \mathrm{~m}$ ) are placed between the upstream target and the liquid scintillators to help separate neutrons coming from the upstream target and those coming from other sources (primarily the downstream target and room-scattered neutrons).

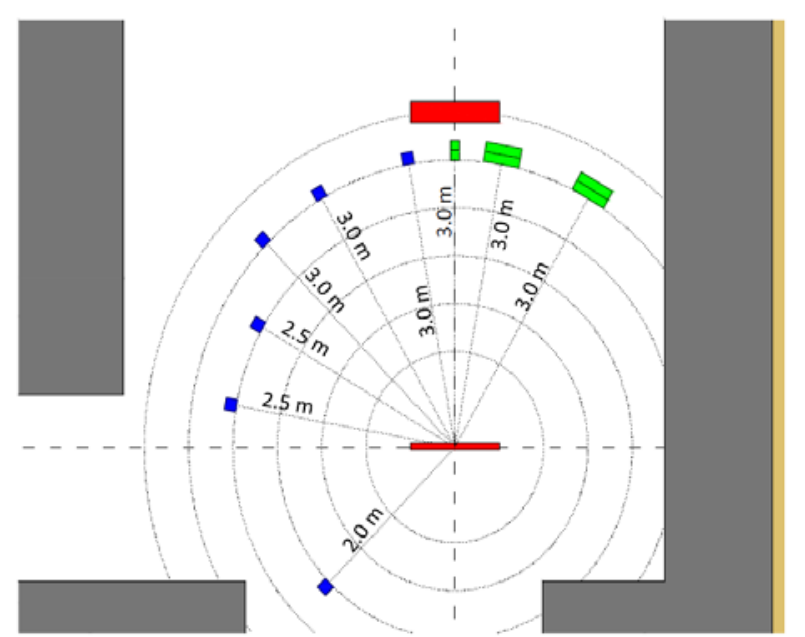

Figure 2. Detector types and locations, as well as relative target thicknesses and locations, for 2016 experiment at BNL.

Among other changes for the experimental run occurring in March 2016 include a second downstream target, as well as an increase in beam species and energies: the front target varies between 20, 40 and 60 $\mathrm{g} / \mathrm{cm}^{2}$ aluminum and the beam species include helium, in addition to iron and protons, at energies of 400 and 800 $\mathrm{MeV} /$ nucleon. The back target is placed $3.5 \mathrm{~m}$ downstream from the front target, and is composed of either aluminum or HDPE with a surface density of 60 $\mathrm{g} / \mathrm{cm}^{2}$. A detailed schematic showing features of the irradiation room is presented in Figure 3, illustrating the locations of the aforementioned targets and detectors with regards to the physical beamline at NSRL. Table 1 summarizes the beam species/energy/shielding configurations used in both the commissioning run and the March 2016 follow-up run.

Table 1 - Summary of experimental setups

\begin{tabular}{|c|c|c|}
\hline Beam Species & Beam Energy (MeV) & Shielding $\left(\mathbf{g} / \mathbf{c m}^{\mathbf{2}}\right)$ \\
\hline Helium $(\mathrm{He})$ & 400 & $\mathrm{Al}: 20,40,60+60$ \\
\hline Hydrogen $(\mathrm{H})$ & 400 & $\mathrm{Al}: 20,40,60+60$ \\
\hline Hydrogen $(\mathrm{H})$ & 800 & $\mathrm{Al}: 20,40,60+60$ \\
\hline Hydrogen $(\mathrm{H})$ & 400 & $\mathrm{Al}: 30$ \\
\hline Hydrogen $(\mathrm{H})$ & 2500 & $\mathrm{Al}: 30$ \\
\hline Iron $(\mathrm{Fe})$ & 400 & $\mathrm{Al}: 20,40,60+60$ \\
\hline Iron $(\mathrm{Fe})$ & 400 & $\mathrm{Al}: 30$ \\
\hline Iron $(\mathrm{Fe})$ & 800 & $\mathrm{Al}: 20,40,60+60$ \\
\hline Iron $(\mathrm{Fe})$ & 1000 & $\mathrm{Al}: 30$ \\
\hline
\end{tabular}

Not present in any of these schematics is the illustration of the thin solid plastic scintillators, all of which cover the faces of the particle detectors. These scintillator paddles are important, for they allow for 
neutron/charged-particle discrimination, and identifying event occurrence (i.e. detection/triggering). These veto paddles are placed facing the target (the liquid scintillators have an additional paddle covering the detector face) - two were used for each neutron EJ-301 scintillators, and six (three in the front and three in the back) for the sodium iodide array. The paddles used in conjunction with the sodium iodide arrays also allow for increased angular dependencies (by focusing on particles triggered by specific configurations of the six paddles) for energy deposition data and measuring particle flight times. Additionally, two scintillating paddles were used in front of the upstream target to help detect incoming beam particles, which allowed for the discrimination of detected events by focusing only on the valid beam particles. Furthermore, these paddles were used within the logic units to set triggers for particle detection within the detectors - by splitting the signal into a real-time signal and a delayed signal, a time gap between the realtime (start) and delayed (stop) is created. This time gap is used to correlate coincidences between these "Start" paddles and the initial signals registered by the paddles in front of the sodium iodide detectors. If a correlation is confirmed, a true signal is registered and the data point is deemed 'valid'.
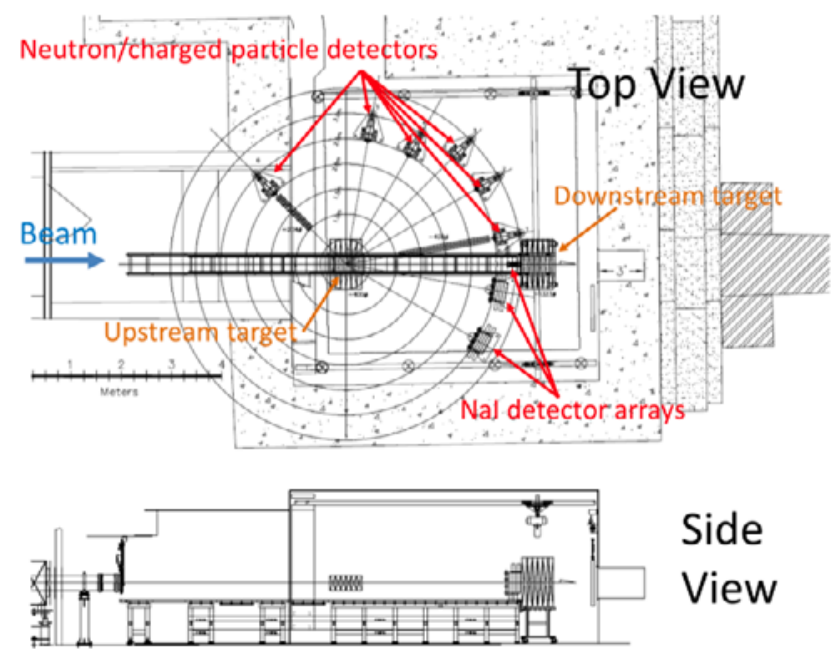

Figure 3. Schematic of experimental setup to be used for thicktarget measurements at NSRL/BNL, illustrating location of beam direction, target location and detector location.

\section{Experimental Analysis}

The analysis process required several steps to produce the relevant particle spectra. The first such step involved the two scintillator paddles in front of the upstream target. The secondary paddle has a slightly smaller active area than the former, and as such by plotting the charge deposition against each other, the "good beam" can be identified. This region can be seen in Figure $4-$ the central oval is the good beam. Figure 4 is an example from the iron $800 \mathrm{MeV} /$ nucleon run on 60 $\mathrm{g} / \mathrm{cm}^{2}$ - these types of plots were used for each set of runs. By focusing on the good beam, extraneous events that set incorrect timing information can be removed, as well as ignoring any particles that were off-axis relative to the beamline.

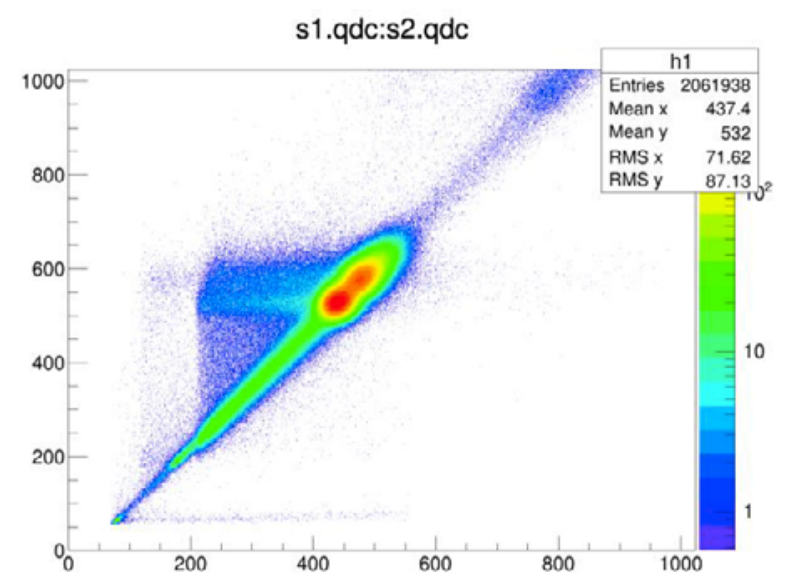

Figure 4. Iron $800 \mathrm{MeV} /$ nucleon charge deposition plot used to identify the good beam. The front paddle (Y-axis) is plotted against the back paddle (X-axis) in terms of channel number.

Once this initial beam cutoff is made, the timing information from the veto paddles is necessary. There are essentially two signals registered by the timing modules for the paddles - a self-time peak that represents the timing coincidence between the veto paddle and the Start paddles, and an overflow peak that represents all of the data when a different detector set the trigger. By further gating the data on the self-time peaks for the front three paddles in front of each sodium iodide array in inclusive disjunction, the data from the sodium iodide arrays is only due to particles who have been born as a result of setting off timing-based triggers in both the Start scintillator paddles (in front of the upstream target) and the veto paddles (immediately in front of the sodium iodide arrays).

The next step in the analysis is charged particle identification. There are two methods used within the sodium iodide array, with varying levels of success. The time-of-flight (ToF) method involves the timing information from the Start paddles compared with the energy deposited within the front sodium iodide detector. An example of a ToF plot from the front $10^{\circ}$ sodium iodide detector is shown in Figure 5. The channel numbers for flight time are inversely related - a higher channel number represents a shorter flight time. Each individual curve represents a specific charged particle ion, and the peak point of each curve represents the "punch-through" point. The punch-through point represents the maximum amount of energy that can be deposited before the particle ranges out in the forward detector. The dynamic energy range of the detector can be summarized by how much of each right leg can be accurately ascribed to a particular isotope. The punchthrough point can be used as a point to calibrate the timing data - converting the channel number into a time scale (nanoseconds) - via the distances between the start paddles, the target (i.e. where the secondary particles are born) and the detector. As can be seen in Figure 5, the left leg (relative to the punch-through point) for each particle species can be cleanly identified, but there is a 
significant overlap of the right legs (as well as extraneous particle background- that is, particles that cannot be segmented out of the relevant data). As such, the use of a second method - comparing energy deposition in each detector - is necessary, because of poor particle identification in that second (right) leg, as well as inconsistencies with the timing data from the veto paddle triggers.

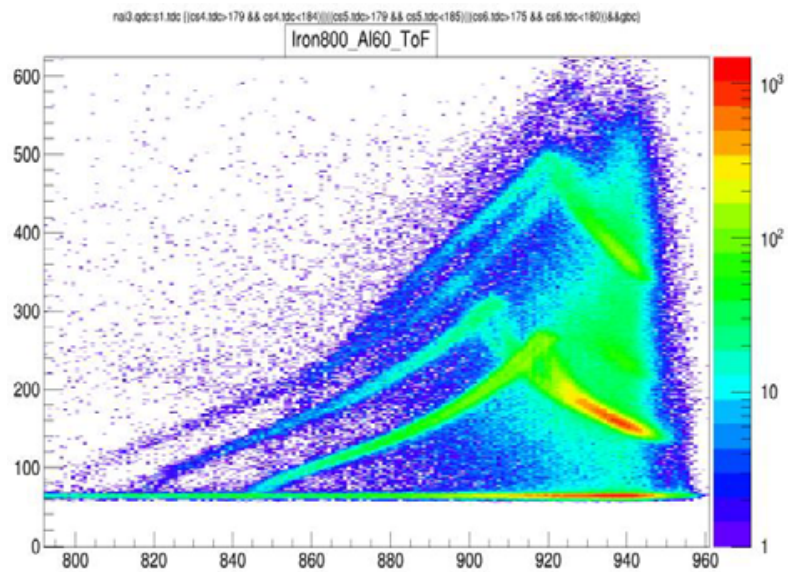

Figure 5. Iron $800 \mathrm{MeV} /$ nucleon on a $60 \mathrm{~g} / \mathrm{cm}^{2}$ Aluminum target in the $10^{\circ}$ sodium iodide detector array. The plot represents timing data (x-axis) plotted against energy deposition (y-axis).

Figure 6 - from the same iron $800 \mathrm{MeV}$ system with the detectors from the $10^{\circ}$ sodium iodide detectors shows the charge deposited in the forward detector ( $y$ axis) against the charge deposited in the back detector ( $x$-axis). A similar pair of "legs" and a "punch-through point" can be seen in Figure 6. The legs represent the energy deposited in each detector - the top leg represents particles that deposited their energy in the front detector whilst the bottom represents particles with enough energy to make it through the front and deposit the remainder in the back. A second calibration point (the first being the punch-through point) is found in the bottom left of Figure 6 - this bright red spot represents zero energy deposition, and by assuming a linear relationship, the zero energy and the punch-through point can be used to convert the channel numbers into energy deposition in $\mathrm{MeV}$.

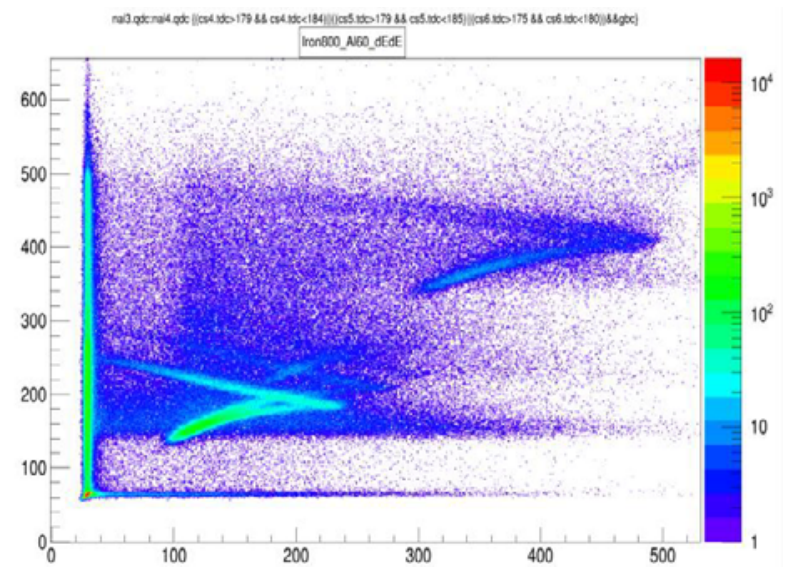

Figure 6. Iron $800 \mathrm{MeV} /$ nucleon on a $60 \mathrm{~g} / \mathrm{cm}^{2}$ Aluminum target in the $10^{\circ}$ sodium iodide detector array. The plot represents energy deposition in the front (y-axis) versus back (x-axis) detector.

Figure 6 has clearer particle identification than the ToF data in Figure 5. Each set of curves represents a unique $Z$ value. In the example provided in Figure 6, three unique $Z=1$ lines can be seen (from bottom to top: proton, deuteron and triton) and a $Z=2$ (helium-4) line. By graphically gating on each set of legs, the specific data corresponding to each particle can be identified. The data is then projected onto the $y$-axis (which is the front detector of each array), creating a particle histogram.

It is important to note that for the $Z=1$ data, there are visible regions of overlap between protonsdeuterons, deuterons-tritons and so on. In an effort to avoid "double counting" (i.e. including deuteron results in a proton spectrum and vice versa), data interpolation in the overlap regions was done. By comparing the ratio of deuterons/protons in other data, an estimation for the expected ratio can be calculated and then used to ensure that the interpolated data is calculated correctly.

As mentioned earlier, the channel numbers were converted to energy deposition using the punch-through point. And using NIST tables as well as the Bethe-Bloch equation (Equation 1), for each particle species the energy deposition can be reverse-calculated into the incoming energy of each particle. Energy deposition is directly correlated with the energy of the incoming particle, and the NIST table with the Bethe-Bloch equation are used to make this calculation [6].

$-\frac{d E}{d x}=\frac{5.08 \times 10^{-31} z^{2} n}{\beta^{2}}\left[\left(\ln \frac{1.02 \times 10^{6} \beta^{2}}{1-\beta^{2}}-\beta^{2}\right)-\ln I_{E V}\right]$

In terms of uncertainty analysis, there were a few sources of error - systematic and statistical. The systematic uncertainties came from uncertainties in the solid angle, detector efficiency and graphic cuts performed on the data. The statistical errors came from the number of measured events. The errors in energy were also determined to be a property of the data as well - the width of the particle species identifiers (the legs from Figures 5 and 6) were determined to best represent the uncertainty in the deposited energy.

\section{Experimental Results}

Depending on the experimental setup (incoming source particle, source energy and shielding), different particles could be identified at the various detectors. Table 2 summarizes what particles could be identified for each setup (and with which sodium iodide array block $-10^{\circ}$ or $30^{\circ}$ ), and the resulting figures are an illustrative example of the calculated results. The italics represent those particles that were detected, but for which a statistically significant amount of data is unavailable. For ease of comparison, only proton results from the $10^{\circ}$ sodium iodide array are presented for each beam species-energy configuration at all three upstream target thicknesses. Additional plots from the proton runs will also be 
presented to show the angular dependence on the data. Figures 7-9 illustrate the proton yield [\#/(source particleMeV-steradian)] on a logarithmic scale plotted against the incoming particle energy.

Table 2 - Summary of particle identification

\begin{tabular}{|c|c|}
\hline Beam Species/Energy [MeV] & Particles (Angle) \\
\hline & Proton (10) \\
& Deuteron (10) \\
Helium (He) 400 & Helium (10) \\
& Proton (30) \\
& Helium-4 (30) \\
\hline \multirow{3}{*}{ Hydrogen (H) 400} & Proton (10) \\
& Deuteron (10) \\
& Proton (30) \\
\hline \multirow{3}{*}{ Hydrogen (H) 800} & Proton (10) \\
& Deuteron (10) \\
& Proton (30) \\
\hline \multirow{3}{*}{ Iron (Fe) 400} & Proton (10) \\
& Deuteron (10) \\
& Triton (10) \\
& Helium-4 (10) \\
& Proton (30) \\
& Deuteron (30) \\
\hline Iron (Fe) 800 & Proton (10) \\
& Deuteron (10) \\
& Triton (10) \\
& Helium (10) \\
& Proton (30) \\
& Deuteron (30) \\
& Helium-4 (30) \\
\hline
\end{tabular}

Figures 7-9 display the detected proton flux at $10^{\circ}$ as a function of their incoming energy for the following incoming species: helium at $400 \mathrm{MeV} /$ nucleon (Fig. 7), iron at $400 \mathrm{MeV} /$ nucleon (Fig. 8), and iron at 800 $\mathrm{MeV} /$ nucleon (Fig. 9).

He 400 - Proton Yield

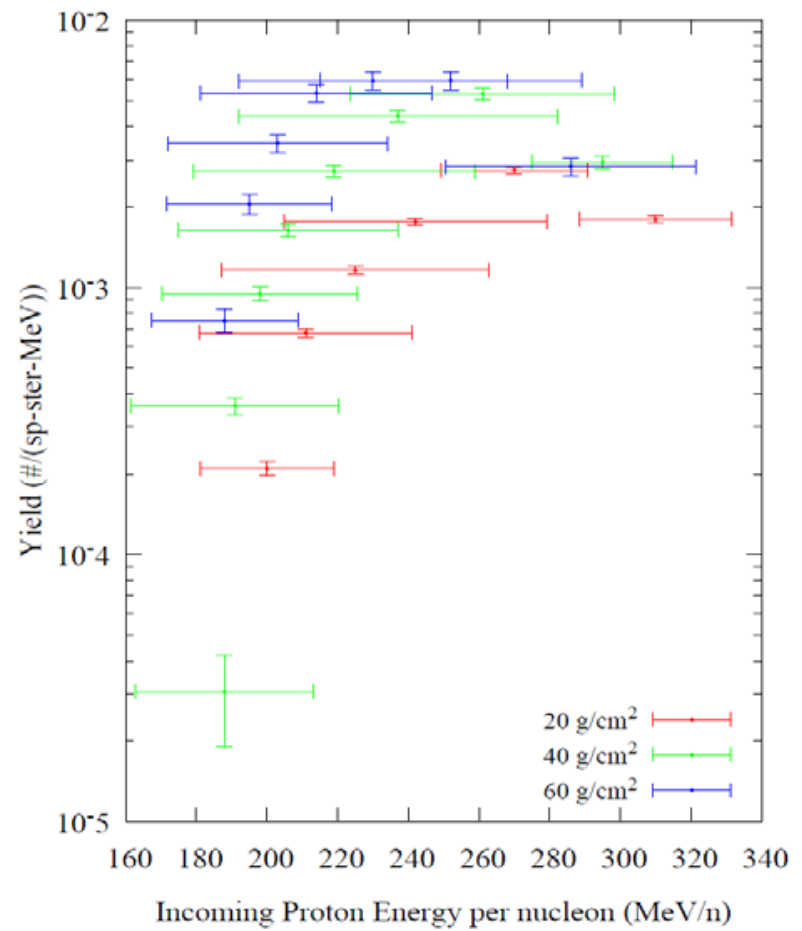

Figure 7. Proton yield from $4-\mathrm{He} 400 \mathrm{MeV} /$ nucleon on varying aluminum shield areal densities.
Fe 400 - Proton Yield

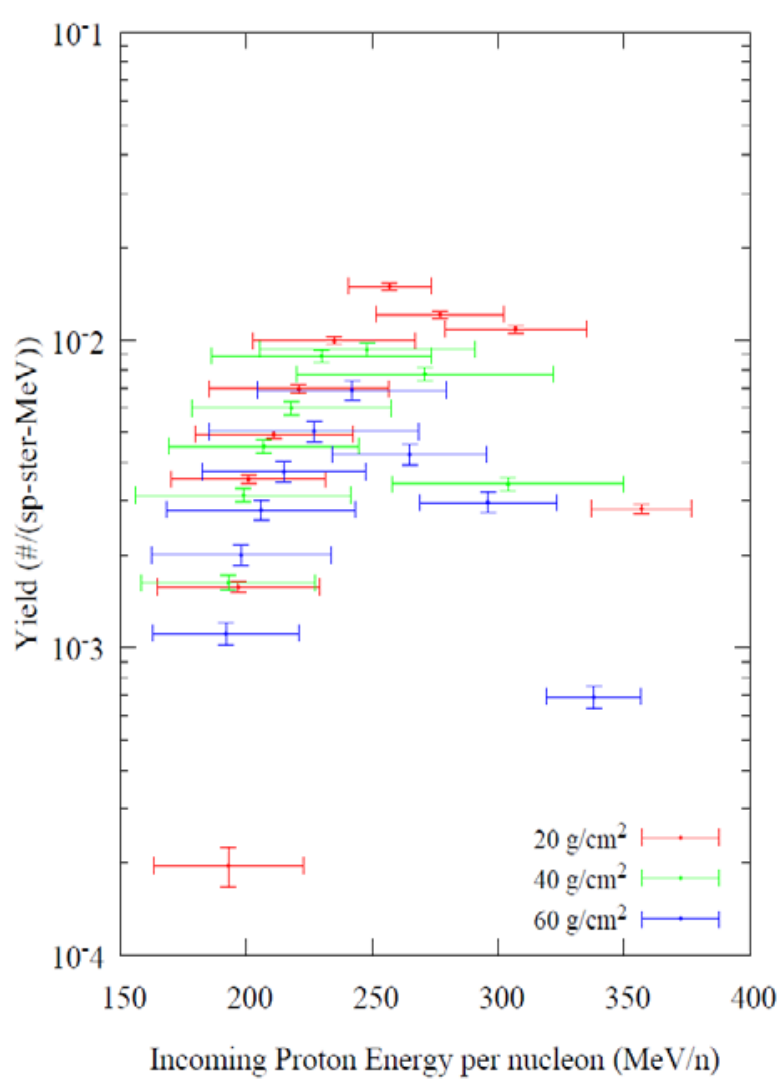

Figure 8. Proton yield from 56-Fe $400 \mathrm{MeV} /$ nucleon on varying aluminum shield areal densities.

Fe 800 - Proton Yield

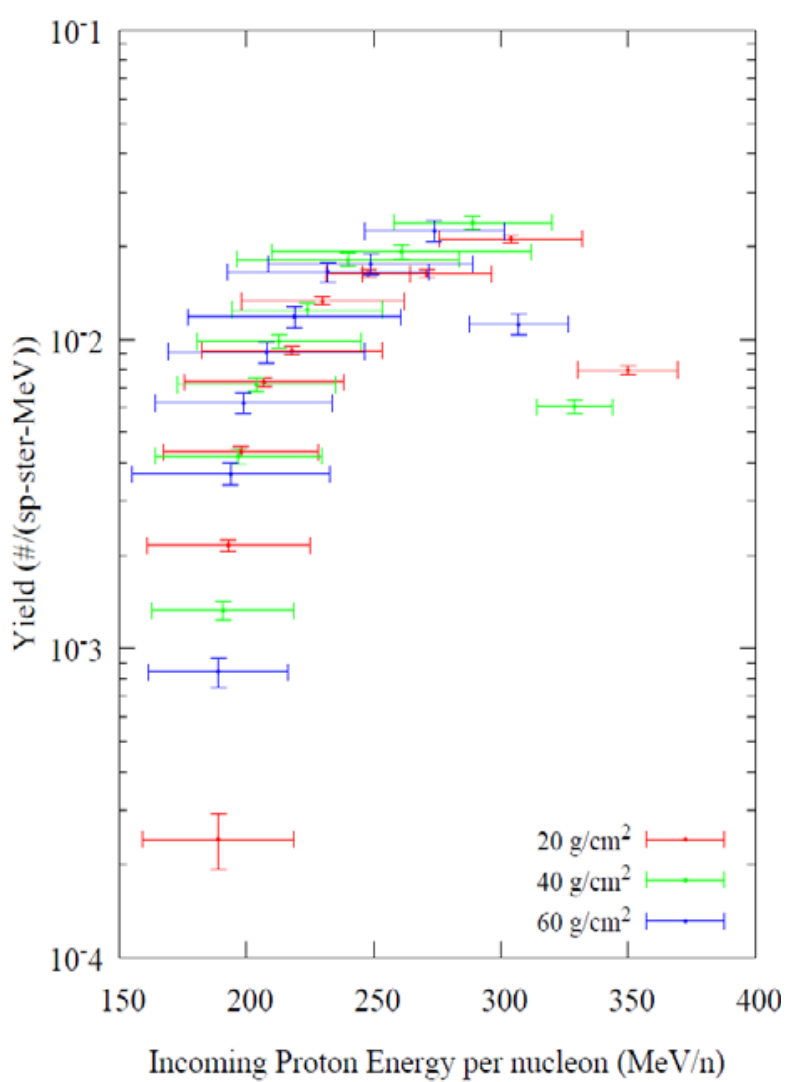

Figure 9. Proton yield from 56-Fe $800 \mathrm{MeV} /$ nucleon on varying aluminum shield areal densities 
The most interesting conclusion from the data presented in Figures 7-9 is that as the areal density of the upstream aluminium target increases, the proton yield (normalized to incoming source particle) actually increases, which seems to support the simulations (Figure 1). Another interesting takeaway is that as the incoming particle energy decreases, so does the associated errors with the calculated yield. These secondary particles are created when the incoming particle undergoes nuclear interactions within the upstream shield, so the statistics at higher energies would be worse, as the physics suggests that less secondary light ions would be created at higher energies. Thus, the statistical errors (both statistical and systematic errors are summed in the illustrated error bars) would be inherently higher as a result of fewer measured events at those energy ranges. The thicker target also inherently would also provide more material for the source particles to traverse through, so it supports the fact that there are higher yields of these light ions at higher shielding thicknesses.

Figures 10-11 show the proton yield from hydrogen nuclei at $400 \mathrm{MeV} /$ nucleon (Fig. 10) and 800 $\mathrm{MeV} /$ nucleon (Fig. 11) for each shielding thickness, comparing the yield at the respective sodium iodide detector blocks $\left(10^{\circ}\right.$ and $\left.30^{\circ}\right)$.
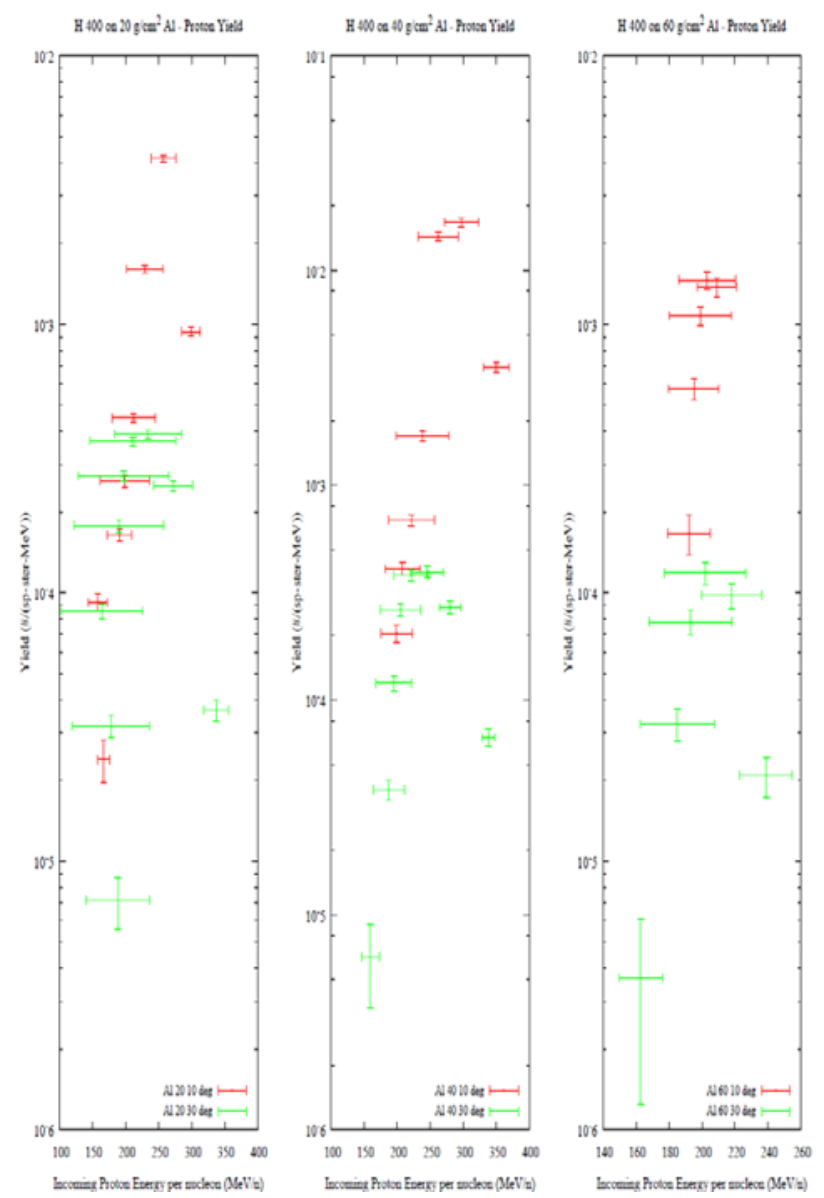

Figure 10. Spatially-dependent proton yields from a 400 $\mathrm{MeV} /$ nucleon upon $20 \mathrm{~g} / \mathrm{cm}^{2}$ (left) $40 \mathrm{~g} / \mathrm{cm}^{2}$ (middle) $60 \mathrm{~g} / \mathrm{cm}^{2}$ (right) aluminum target.
Generally, there is increased yield at every thickness for the $10^{\circ}$ detector block as compared to the $30^{\circ}$ block. Considering that the light ions are created from beam interacting within the shielding, it is presumed that most are initially produced along the beamline, so the closer the detector is to the center of the upstream and downstream targets, the higher the expected yield. The breakup of the projectile is also a source of these light ions, and as such the projectile fragments maintain the projectile's momentum in the forward direction. In conjunction with trends from Figures 7-9, errors tend to decrease with incoming particle energy, and that the thicker shielding results in higher yields. Furthermore, the yields from increased thicknesses result in more of the data being in lower energy ranges than the thinner shields.
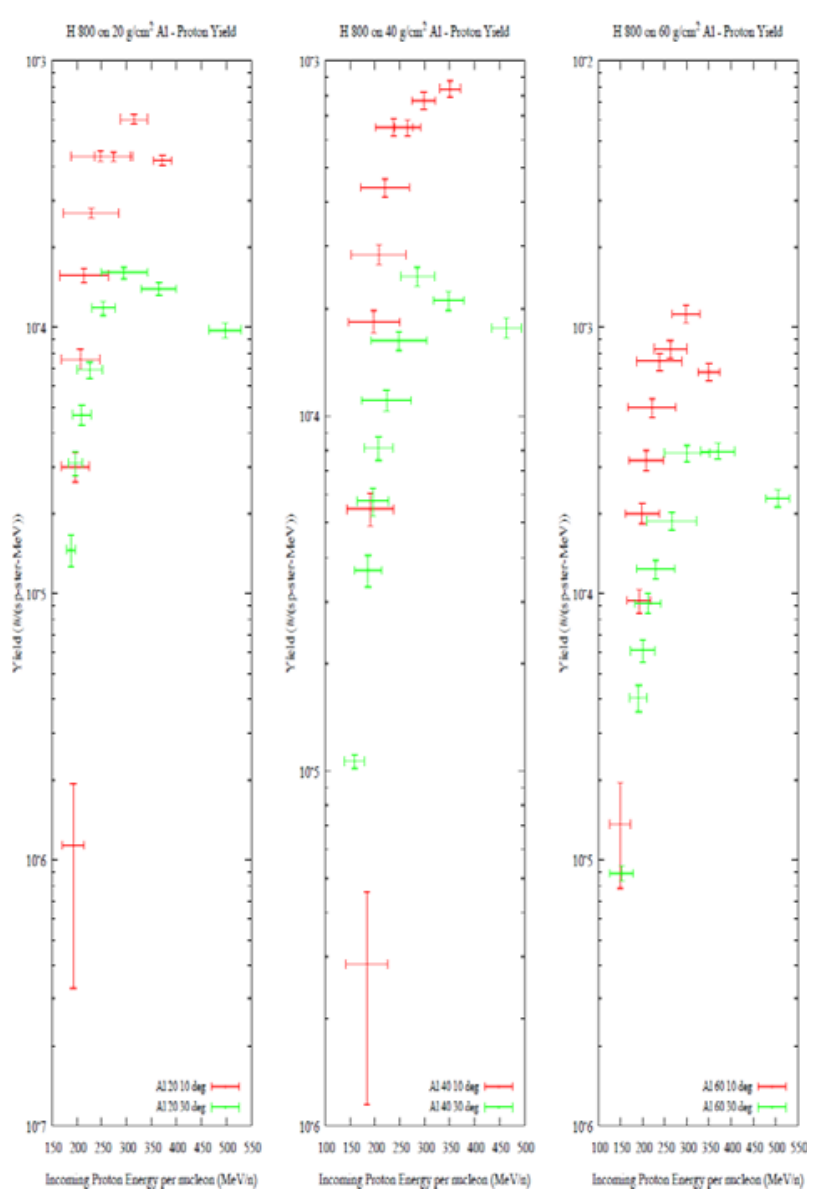

Figure 11. Spatially-dependent proton yields from a 800 $\mathrm{MeV} /$ nucleon upon $20 \mathrm{~g} / \mathrm{cm}^{2}$ (left) $40 \mathrm{~g} / \mathrm{cm}^{2}$ (middle) $60 \mathrm{~g} / \mathrm{cm}^{2}$ (right) aluminum target.

There are two sources of these secondary particles - breakup of the source particle, and breakup of the target. Figures 10 and 11 suggest that the wider the angle, the less yield measured and that even when the incoming particle energy [significantly] increases, a majority of the secondary particles come at energies lower than the source particle energy. This is primarily due to energy loss through the target after production. 


\section{Future Work}

The next experiment is scheduled to take place in December 2016 at the NASA Space Radiation Laboratory at Brookhaven National Laboratory with the intent of adding new beam species and energies to the already-studied systems (Table 1). Table 3 summarizes the new beam species/energies and targets that are expected to be studied in the December 2016 run.

Further analysis is expected within this current data set as well - there are certain light ions for which there is data (the italicized species in Table 2) but it needs further graphical analysis to remove background data, and the errors associated with the incoming particle energy calculations will be reduced.

Table 3 - Upcoming (Dec. 2017) experimental setups

\begin{tabular}{|c|c|c|}
\hline Beam Species & Beam Energy (MeV) & Shielding $\mathbf{( g / \mathbf { c m } ^ { 2 } )}$ \\
\hline Helium $(\mathrm{He})$ & 800 & $\mathrm{Al}: 20,40,60+60$ \\
\hline Helium $(\mathrm{He})$ & 1500 & $\mathrm{Al}: 20,40,60+60$ \\
\hline Silicon $(\mathrm{Si})$ & 400 & $\mathrm{Al}: 20,40,60+60$ \\
\hline Silicon $(\mathrm{Si})$ & 800 & $\mathrm{Al}: 20,40,60+60$ \\
\hline Silicon $(\mathrm{Si})$ & 1500 & $\mathrm{Al}: 20,40,60+60$ \\
\hline Carbon $(\mathrm{C})$ & 400 & $\mathrm{Al}: 20,40,60+60$ \\
\hline Carbon $(\mathrm{C})$ & 800 & $\mathrm{Al}: 20,40,60+60$ \\
\hline Carbon $(\mathrm{C})$ & 1500 & $\mathrm{Al}: 20,40,60+60$ \\
\hline Iron $(\mathrm{Fe})$ & 1500 & $\mathrm{Al}: 20,40,60+60$ \\
\hline
\end{tabular}

\section{Summary}

A series of thick-target experiments has been designed to aid NASA interests in deep-space manned missions by providing experimental data to assist with improving the transport models via uncertainty quantification. A commissioning experiment at the NSRL facility at BNL was performed in May 2015, with the preliminary results presented here. The methodology was studied and improved upon from the commissioning run, and the first set of data was taken in March 2016 runs. Double differential thick-target yields were calculated (as a function of incoming particle energy) for the light ions of interest. Between the March 2016 run and future runs at NSRL, the chosen beam species and energies will - as a whole - simulate the important components of the primary GCR field.

\section{Acknowledgments}

This work was supported by Grants NNX12AL51A and NNX15AD89A from the United States National Aeronautics and Space Administration.

\section{References}

1. D.F. Smart, M.A. Shea, Handbook on Geophysics (1985)

2. T. Slaba, 2015 Advanced Radiation Protection EOY Review, 1-15 (2015).

3. L. Heilbronn, R.S. Cary, M. Cronqvist, F. Deak, K. Frankel, A. Galonsky, K. Holabird, A. Horvath, A.
Kiss, J. Kruse, and others, Nucl. Sci. Eng., 132, 1-15 (1999)

4. P.-E. Tsai, "Study of secondary particles produced from heavy-ion interactions," Ph.D. dissertation, Dept. of Nucl. Eng., Univ. of TN, Knoxville, TN (2015)

5. T. Nakamura, L. Heilbronn, Handbook on Secondary Particle Production and Transport by High-Energy Heavy Ions (2006)

6. International Commission on Radiation Units and Measurements, ICRU Report 49, Stopping Powers and Ranges for Protons and Alpha Particles (1993) 